\section{Risk stratification of prostate cancer: integrating multiparametric MRI, nomograms and biomarkers}

\author{
Matthew J Watson', Arvin K George', Mahir Maruf', Thomas P Frye', Akhil Muthigi', \\ Michael Kongnyuy', Subin G Valayil' \& Peter A Pinto*,1
}

Accurate risk stratification of prostate cancer is achieved with a number of existing tools to ensure the identification of at-risk patients, characterization of disease aggressiveness, prediction of cancer burden and extrapolation of treatment outcomes for appropriate management of the disease. Statistical tables and nomograms using classic clinicopathological variables have long been the standard of care. However, the introduction of multiparametric $\mathrm{MRI}$, along with fusion-guided targeted prostate biopsy and novel biomarkers, are being assimilated into clinical practice. The majority of studies to date present the outcomes of each in isolation. The current review offers a critical and objective assessment regarding the integration of multiparametric MRI and fusion-guided prostate biopsy with novel biomarkers and predictive nomograms in contemporary clinical practice.

First draft submitted: 8 April 2016; Accepted for publication: 7 June 2016; Published online: 12 July 2016

Currently, prostate cancer ( $\mathrm{PCa}$ ) screening relies on serum prostate-specific antigen (PSA) and a digital rectal examination (DRE). However, these tests suffer from limited sensitivity and specificity, resulting in unnecessary biopsies [1,2]. Classically, an elevated PSA or suspicious DRE triggers a systematic 12-core transrectal ultrasound-guided prostate biopsy (SBx). SBx may result in underdetection or undergrading of PCa potentially causing treatment delay resulting in patient morbidity. Conversely, SBx may also diagnose otherwise clinically insignificant cancers, often leading to subsequent overtreatment and also increasing patient morbidity [3]. For this reason, the US Preventive Services Task Force recommends against routine PSA screening [4]. However, the effect of decreased screening has resulted in diminished detection of early stage prostate cancer, of which the effects on mortality are yet unknown [5]. Instead of indiscriminate and inappropriate PSA testing and prostate biopsy, the use of available and emerging tools may provide additional insight into men at greatest risk for harboring clinically significant (CS) disease (herein defined as $\geq$ Gleason score (GS) $3+4=7$, unless otherwise specified).

MRI has improved the detection of CS PCa due to its anatomic detail, emerging accessibility, and addition of functional data [6-8]. MRI of the prostate is optimized for the detection of CS PCa with negative and positive predictive values exceeding 90\% [9-11]. Inclusion of MRI in the diagnostic pathway has initiated a change in how urologists recognize and manage PCa. Multiparametric MRI (mpMRI), consisting of T2-weighted (T2W), axial diffusion-weighted imaging (DWI) with apparent diffusion coefficient (ADC) mapping and dynamic contrast-enhanced (DCE) sequences, improves upon prostatic tumor detection, localization and sensitivity for distinguishing CS disease (Figure 1). As a result, it may potentially serve as a noninvasive tool which better risk stratifies men who are at risk for PCa.

'Urological Oncology Branch, National Cancer Institute, NIH, Bethesda, MD, USA

*Author for correspondence: Tel.: +1 301496 6353; pintop@mail.nih.gov

\section{KEYWORDS}

- biomarkers

- multiparametric MRI

- nomograms $\bullet$ prostate

- prostate cancer 
In 2012, a group of MRI experts from the European Society of Urogenital Radiology (ESUR) developed clinical guidelines for mpMRI of the prostate based on literature evidence and expert opinion agreement [12]. The Prostate Imaging Reporting and Data System (PI-RADS) appears to have good diagnostic accuracy in PCa detection in patients for whom PCa is suspected [6]. mpMRI data are presented in a regulated reporting scheme, consisting of a PI-RADS score. This score communicates the possibility of cancer risk, aggression, location and probability of extraprostatic disease along with relevant supplementary findings. However, although PI-RADS standardizes PCa lesion scoring, the additional training necessary to properly interpret the mpMRI sequences to assign a score may be a barrier to mpMRI's widespread use.

mpMRI in combination with MRI/ultrasound fusion-guided targeted biopsy (FBx) has emerged as a promising alternative to SBx [13-16]. mpMRI's indications are rapidly expanding given the more accurate grading and staging of disease compared with the current standard of care $[17,18]$. Though confidence in the negative predictive value of mpMRI and FBx has been growing, it has its limitations with presence of Gleason $\geq 7$ PCa noted in 2.3-20\% of patients with a negative study [19-21]. This can be due to $\mathrm{MR}$ reader and biopsy experience, difference in scan quality and patient selection for MRI. Additionally, the cost-effectiveness, how to best integrate this information into clinical practice and patient satisfaction all remain significant areas of research.

Given the need to distinguish between indolent and aggressive $\mathrm{PCa}$ for patient counseling and treatment, clinicians have made efforts to create evidence-based algorithms that use specific patient's clinicopathological variables. In an effort to reduce the overdiagnosis and overtreatment of PCa, a number of methodologies, including serum biomarkers, statistical based predictive algorithms and recently mpMRI, have been used to more accurately assess the risk of PCa when compared with PSA and DRE alone [22-25]. Examples of these tools include statistical models such as predictive tables and nomograms [26]. Herein, we present a clinical perspective of the incorporation of mpMRI with existing tools including prognostic nomograms and biomarkers, in the risk stratification of $\mathrm{PCa}$.

\section{Biomarkers \& mpMRI for risk stratification}

Traditionally, total PSA has been used to screen men for PCa. Total PSA shows good sensitivity, but low specificity and a moderate AUC $(83.4 \%, 38.9 \%$ and 0.678 , respectively) in screening [27]. As the pitfalls of PSA testing have become apparent, novel biomarkers have been proposed to address the less than optimal performance characteristics of the PSA test. These biomarkers improve upon the identification of men at risk to harbor CS disease and should be

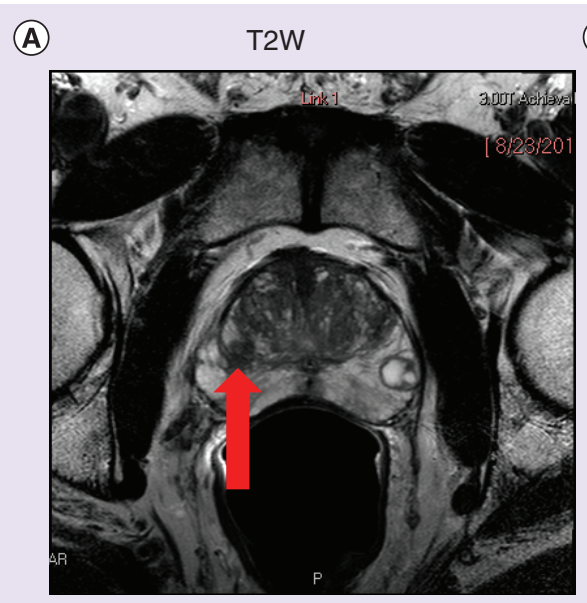

(B)

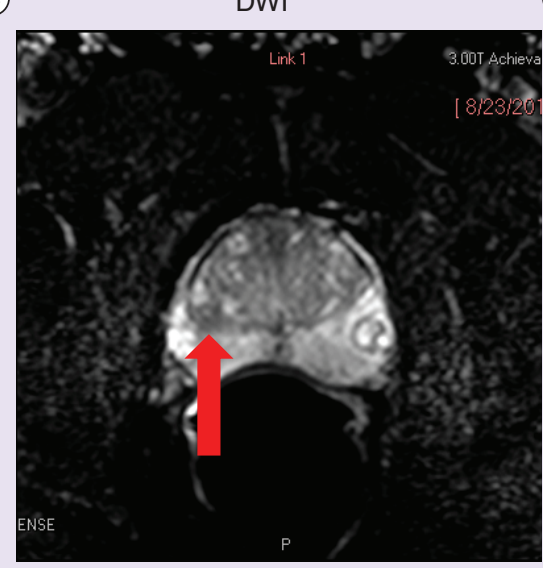

(C)

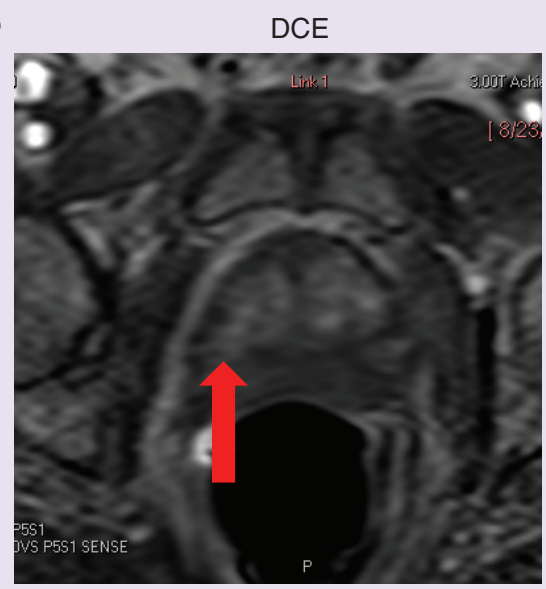

Figure 1. Multiparametric MRI of prostate from 66-year-old male with history of Gleason score $3+4=7$ on systematic 12 -core transrectal ultrasound-guided prostate biopsy, subsequently upgraded to Gleason score $4+4=8$ following multiparametric MRI and fusion-guided targeted biopsy. Low signal intensity on T2W, restricted diffusion on DWI and focal enhancement on DCE reveal $0.7 \mathrm{~cm}$ lesion on right mid transitional zone (arrows).

DCE: Dynamic contrast-enhanced; DWI: Diffusion-weighted imaging; T2W: T2-weighted. 
Table 1. Biomarkers used to identify patients with prostate cancer.

\begin{tabular}{|c|c|c|c|c|}
\hline Test characteristics & Total PSA & PCA3 & Prostate Health Index & 4-Kallikrein score \\
\hline Site of derivation & Blood & Urine & Blood & Blood \\
\hline Paramater test measures & $\begin{array}{l}\text { Enzyme produced } \\
\text { exclusively by prostate cells }\end{array}$ & $\begin{array}{l}\text { Overexpression of } D D 3 \\
\text { gene (seen in } 95 \% \text { of } \mathrm{PCa} \text { ) }\end{array}$ & $\begin{array}{l}\text { Combines three forms of } \\
\text { PSA enzyme (total PSA, } \\
\text { free PSA, [-2]pro-PSA) }\end{array}$ & $\begin{array}{l}\text { Combines four enzymes } \\
\text { (total PSA, free PSA, intact } \\
\text { PSA, human KLK2) }\end{array}$ \\
\hline AUC for predicting $\mathrm{PCa}$ & 0.678 & 0.75 & 0.7 & 0.821 \\
\hline
\end{tabular}

recommended for biopsy and include PCA3, prostate health index (PHI) and 4-Kallikrein (4K) score (Table 1). PCA3 has been shown to have a lower sensitivity (73\%), but higher specificity and AUC (65\% and 0.75 , respectively) for prostate cancer detection [28]. Meta-analysis of the PHI shows a similar utility of PSA for PCa screening with sensitivity, specificity and area under the receiver operator characteristic curve (AUC) of $85 \%, 45 \%$ and 0.7 , respectively [29]. Finally, $4 \mathrm{~K}$ score showed the greatest ability to predict those with CS PCa with an AUC found to be 0.821 in a prospective, multi-institutional trial [30]. The use of mpMRI also improves risk stratification, but with the additional benefit of anatomic data (i.e., size, location, multifocality). This allows direct targeting of suspicious lesions with FBx. Imaging has been shown to be of benefit over PSA alone, but to date few studies directly compare the performance of mpMRI to commonly used biomarkers [31].

One such study sought to characterize the diagnostic accuracy of Total PSA, PCA3, PHI and mpMRI in men with prior negative prostate biopsies [32]. One hundred seventy men were analyzed and the results revealed that mpMRI contributed the most in identifying cancer on repeat biopsy (AUC: 0.936). This AUC for mpMRI was significantly higher than the PHI and PCA3 combined AUC. Of note, men identified with anterior lesions were excluded from this study and SBx was performed. It is likely that if patients with anterior lesions were included, and FBx were implemented, the performance of mpMRI would be of even more benefit.

The same group compared the ability of PHI, PCA3 and mpMRI in predicting CS PCa in men eligible for active surveillance (AS), but elected to undergo radical prostatectomy [33]. They defined CS PCa as those cancers that did not meet the European Randomized Study of Prostate Cancer definition of insignificant disease (Table 2). A total of 100 patients met inclusion criteria. Both PHI and mpMRI were found to significantly increase the AUC in predicting which men harbored CS disease $(\mathrm{p}<0.01)$. Furthermore, decision curve analysis showed that the multivariable model including mpMRI had the highest net benefit.

Other studies have examined the clinical utility of combining biomarker and mpMRI data in identifying patients harboring PCa. One group evaluated PCA3 in combination with mpMRI in men with prior negative biopsies [34]. Using PI-RADSv1, they found that all men with a PI-RADS III (moderate suspicion) score, using who had biopsy-proven PCa had a PCA3 greater than 35 . Thus, PCA3 could potentially help differentiate which patients with moderately suspicious prostate lesions should proceed with prostate biopsy. A similar study found that men with lesions suspicious for prostate cancer on mpMRI had significantly higher PCA3 scores than men with no lesions (52 vs 21; p < 0.001) [35]. They concluded that PCA3 could potentially be used as a screening tool to determine which men should receive an mpMRI.

\section{Risk calculators \& mpMRI}

The Prostate Cancer Prevention Trial Risk Calculator 2.0 (PCPTRC) is a commonly used, externally validated, prediction tool for the assessment of a man's risk of PCa [23,24]. The PCPTRC was constructed from the placebo arm of the Phase III Prostate Cancer Prevention Trial. The variables used to build this model were age at biopsy, race, DRE findings, serum PSA and biopsy history [36]. The risk profile of the unscreened population included patients who were $\geq 70$ years old ( $47 \%$ of patients) with a median PSA of $1.5 \mathrm{ng} / \mathrm{ml}$. As a result, inconsistent performance has been observed across various patient cohorts. Area under the receiver operator characteristic curves (AUC) of PCPTRC for cancer detection have ranged between 0.562 and 0.744 in studies that applied the risk calculators in different patient populations [37-42]. A multinational study of ten combined patient cohorts assessed the performance of the PCPTRC for predicting CS disease. The study included eight 
Table 2. Definitions of clinically insignificant prostate cancer on whole mount pathology.

\begin{tabular}{|c|c|c|c|c|}
\hline Definition & Volume & Gleason pattern & Gleason score & Pathological stage \\
\hline $\begin{array}{l}\text { European randomized study of prostate } \\
\text { cancer }\end{array}$ & $\begin{array}{l}\text { Total tumor: } \leq 2.5 \mathrm{~cm}^{3} \\
\text { Index tumor: } \leq 1.3 \mathrm{~cm}^{3}\end{array}$ & No GP 4 or 5 & Gleason score $\leq 6$ & Organ confined (pT2) \\
\hline Epstein & $\leq 0.5 \mathrm{~cm}^{3}$ & No GP $4^{\dagger}$ or 5 present & Gleason score $\leq 6$ & Organ confined (pT2) \\
\hline
\end{tabular}

screening cohorts and two cohorts of patients referred for biopsy. The AUC for high-grade cancer calculated by the PCPTRC ranged from 0.639 to 0.739 in the referral-based population. This less than optimal range of AUCs may be due to the cohort from which the calculator was derived, which may not represent all patient populations. As such, general application for the prediction of CS cancer in all populations may be limited. Furthermore, the ten combined cohort AUC was only a minor improvement to PSA alone ( 0.746 vs $0.715 ; \mathrm{p}<0.0001)$ [41]. The use of a model with these limitations may produce risk estimates which result in unnecessary biopsy or missed CS disease.

mpMRI has been shown to outperform other clinicopathological covariates such as age, PSA and prostate volume in predicting CS disease [43-45]. Imaging with mpMRI may improve the assessment of PCa risk as demonstrated by both the high sensitivity and negative predictive value for detecting PCa [46]. Additionally, mpMRI has demonstrated value in populations with a higher baseline risk of CS PCa [47]. A recent study by Salami et al. directly compared the performance of mpMRI with the PCPTRC for detecting high Gleason pattern cancer and CS disease. The study defined CS PCa those cancers which did not meet the Epstein definition of insignificant disease on whole mount pathology (Table 2) [48]. Suspicious lesions were scored using the PI-RADSv1 system, and were compared with risk calculated by the PCPTRC [12]. Of the 175 patients referred for biopsy due to elevated PSA, abnormal DRE or suspicious lesion on MRI, $47.4 \%$ of those undergoing FBx were diagnosed with high Gleason pattern disease. In comparison, the PCPTRC underestimated the risk of high Gleason pattern disease (20.2\%). An AUC of 0.676 was reported using the PCPTRC model, while mpMRI was found to have an AUC of 0.769 ( $p=0.091)$. Furthermore, when the Epstein definition $[48,49]$ was applied to the mpMRI model, the AUC was calculated as 0.812 , which was significantly higher than the PCPTRC model ( $p=0.008)$ [50]. As such, the addition of mpMRI as a risk calculator covariate may improve upon the detection of CS prostate cancer. Although the PCPTRC has been used to address the overdiagnosis of $\mathrm{PCa}$, its shortcomings may result in a failure to adequately stratify patients at risk for harboring CS disease. Therefore, mpMRI may have an emerging role in the assessment of PCa risk by serving as an adjunct to existing risk calculators and by identifying patients who should require biopsy [51].

\section{Role of mpMRI in active surveillance}

Active surveillance (AS) of PCa is aimed at delaying or completely avoiding treatment until disease progression, while preserving the option of curative therapy when necessary [52,53]. Carefully selected patients undergo serial PSA testing and interval SBx to identify an increase in volume of disease or GS. Classic clinical parameters, such as PSA kinetics, offer limited benefit in defining disease progression and predicting biopsy reclassification during AS [54]. Furthermore, surveillance biopsies tend to detect high numbers of clinically insignificant disease, while simultaneously missing relevant CS cases [55,56]. mpMRI and FBx can improve upon the status quo in three ways: improve classification of truly clinically insignificant disease with mpMRI, confirm AS candidacy with FBx and monitor disease progression with combined serial mpMRI and FBx $[57,58]$.

mpMRI is highly sensitive, with negative predictive values approaching over $90 \%$ for CS cancers [19,59]. Additionally, MRI-assigned lesion suspicion scores (low, moderate, high suspicion) strongly correlate with cancer detection rates and biopsy [45]. A study of 125 patients with low-suspicion prostate lesions found on mpMRI demonstrated a low incidence of CS disease as confirmed via FBx. 107 (88\%) patients either had no cancer or clinically insignificant disease [60]. Thus, either absence of suspicious lesions and/or presence of only low suspicion lesions could serve as useful criteria in patient selection for AS. However, mpMRI has been shown to miss $9 \%$ of high-grade cancers [61]. 
As such, these studies may be biased in favor of mpMRI as FBx, not whole mount pathology, were used as an end point. Furthermore, in a study that did use whole mount pathology as an end point, Turkbey et al. produced a study on 133 patients who underwent mpMRI studies prior to radical prostatectomy (RP) [62]. mpMRI was superior for predicting AS eligibility relative to conventional clinical scoring systems (D'Amico, Epstein and Cancer of the Prostate Risk Assessment [CAPRA] systems) (Table 3), with a sensitivity of $93 \%$, positive predictive value of $57 \%$, and overall accuracy of $92 \%$ $(\mathrm{p}<0.005)$.

Accurate risk classification of clinically insignificant disease is essential to ensure optimal outcomes on AS. mpMRI paired with FBx, may better serve to provide histologic confirmation of AS candidacy. Hu et al. retrospectively examined 113 patients who met Epstein criteria and were enrolled in an AS protocol [63]. Confirmatory FBx resulted in reclassification of 41 men (36\%), either due to diagnosis of GS $\geq 7$ or presence of high volume GS 6 disease. Men with high suspicion targets on imaging (UCLA scoring system; scale: $1-5)$ were reclassified $45-100 \%$ of the time (grade 4: 45\%, grade 5: 100\%; $\mathrm{p}=0.001$ ). Similarly, Stamatakis et al. found 29\% (25/85) of patients placed on AS using Epstein criteria to no longer be eligible for AS after mpMRI and confirmatory FBx [64]. Number of lesions, lesion density and highest MRI lesion suspicion (scale: low, moderate, high) remained the significant mpMRI parameters for reclassification. Recently a prospective comparison of $\mathrm{FBx}$ versus $\mathrm{SBx}$ for detecting CS PCa in patients on AS was completed [65]. GS 7 cancers were found in $19 / 72$ patients (26\%), with $37 \%$ of cases detected by FBx alone, as compared with only $11 \%$ detected by SBx alone. Furthermore,
FBx was greater than six-times more likely to yield a core positive for GS 7 cancer compared with SBx ( $25 \%$ of 141 cores vs $4 \%$ of 874 cores; $\mathrm{p}<0.001)$. Importantly, MRI suspicion level (Likert scale: 1-5) remained an independent predictor for CS PCa on multivariate analysis (OR: 3.6; $\mathrm{p}<0.001$ ). Therefore, mpMRI and FBx has the potential to play a vital role in the confirmation and reclassification of patients that are initially placed on AS.

Serial mpMRI studies, coupled with limited and strategic re-biopsy utilizing fusion techniques, look promising as a tool for accurate surveillance of patients on AS protocols. Utilizing mpMRI as a reference, current platforms have the ability to electronically track specific sites within the prostate, allowing the user to precisely return to previously positive areas for serial resampling over time [66-69]. Walton Diaz et al. examined the utility of acquiring serial mpMRI and fusion biopsies in AS patients to determine GS progression [70]. They found that stable findings on mpMRI, based on MRI suspicion level (low, moderate, high), largest lesion diameter and number of lesions were associated with GS nonprogression. Importantly, the number needed to biopsy to detect one GS progression was 8.74 patients for $S B x$ versus 2.9 patients for FBx. These early studies offer support for the use of serial MRI and FBx with limited number of cores to monitor patients placed on AS.

Initial work examining the utility of mpMRI and $\mathrm{FBx}$ in AS is promising. However, there currently is no consensus on how to accurately define progression of disease on mpMRI and only recently has a standardized scoring system, Prostate Imaging Reporting and Data System version 2 (PI-RADSv2), become widely adopted [71]. Additionally, it has yet to be determined prospectively if serial mpMRIs can

Table 3. Rule-based criteria for predicting active surveillance eligibility at biopsy.

\begin{tabular}{|c|c|c|c|c|c|c|}
\hline Criteria & Age & PSAL/PSAD & GP & $\begin{array}{l}\text { Positive biopsy cores } \\
\text { (n) }\end{array}$ & $\begin{array}{l}\text { Cancer per } \\
\text { positive core }\end{array}$ & Clinical stage \\
\hline Epstein & Not applicable & $\operatorname{PSAD}(\mathrm{ng}):<0.15$ & No GP 4 or 5 & $\begin{array}{l}<3 \text { positive cores } \\
\text { (from sextant biopsy) }\end{array}$ & $\begin{array}{l}<50 \% \text { cancer per } \\
\text { core }\end{array}$ & T1c (organ confined) \\
\hline CAPRA $^{\dagger}$ & $\begin{array}{l}0 \mathrm{pt}:<50 \text { years } \\
1 \mathrm{pt}: \geq 50 \text { years }\end{array}$ & $\begin{array}{l}\text { PSAL }(\mathrm{ng} / \mathrm{ml}): \\
0 \mathrm{pt}: 2.0-6.0 \\
1 \mathrm{pt}: 6.1-10.0 \\
2 \mathrm{pt}: 10.1-20.0\end{array}$ & $\begin{array}{l}\text { Primary/secondary GP: } \\
0 \mathrm{pt}: 1-3 / 1-3 \\
1 \mathrm{pt}: 1-3 / 4-5 \\
2 \mathrm{pt}: 4-5 / 1-5\end{array}$ & Not applicable & $\begin{array}{l}0 \mathrm{pt}:<34 \% \\
1 \mathrm{pt}: \geq 34 \%\end{array}$ & $\begin{array}{l}0 \text { pt: T1/T2 (organ } \\
\text { confined) } \\
1 \text { pt: T3a (extracapsular } \\
\text { extension) }\end{array}$ \\
\hline
\end{tabular}


circumvent the need for biopsy, or whether an FBx limited to mpMRI targets is sufficient. The present evidence supports the use of mpMRI and $\mathrm{FBx}$ to provide confidence in ruling out CS disease. Currently, however, serial MRI and both $\mathrm{SBx}$ and $\mathrm{FBx}$ are necessary to detect progression on surveillance.

\section{Nomograms for staging}

Accurate staging of $\mathrm{PCa}$ is required for risk stratification and determination of treatment options [72]. Stages are assigned as to whether or not the PCa is organ confined, and if not, stratified by local or distant invasion/involvement. Locally advanced disease includes extracapsular extension (ECE), seminal vesicle invasion (SVI) or local pelvic structures, whereas distant disease reflects lymph node involvement (LNI), bone or visceral organ metastasis [73-75]. Predictive staging nomograms allow the urologist to prognosticate clinical outcomes for the patient [76]. The Partin tables are the classic algorithm that combines clinical stage, preoperative serum PSA and biopsy Gleason grade to predict pathological stage. However, mpMRI is increasingly being utilized as a parameter in the development of nomograms informing disease stage.

\section{- ECE}

Patients with locally advanced disease, specifically ECE, are much more likely to experience inferior oncologic outcomes [77,78]. The incidence of ECE is high, being identified in $26 \%$ of radical prostatectomy patients [79]. When ECE is suspected during RP, the surgical approach may be adjusted in an effort to reduce the likelihood of positive margins $[80,81]$. As such, proper preoperative identification allows surgeons to best plan surgical approaches to optimize oncologic outcomes.

Recently, mpMRI alone has been shown to have low sensitivity (48.7\%), but moderate specificity $(73.9 \%)$ for detecting ECE as determined by final pathology [82]. While the low sensitivity of mpMRI on its own can be problematic, mpMRI is still attractive as an added parameter in creating predictive models as it is the only covariate that is able to provide detailed anatomical and functional information. A recent study compared classic clinicopathological nomograms to a nomogram that integrated these with mpMRI findings for the prediction of ECE [83]. Multivariate analysis revealed PSA, greatest percent cancer in all biopsy cores and
mpMRI findings were all significant predictors of ECE ( $p<0.001$ to $p=0.001)$. However, the nomogram utilizing mpMRI discriminated significantly better than the clinicopathological based nomogram (AUC: 0.838 vs 0.722 ; $\mathrm{p}=0.022)$. This study was the first to provide evidence for the utility of mpMRI findings as a parameter in nomograms predicting ECE.

A similarly designed study provided a head-to-head comparison between the performance of the 2001 Partin tables, Memorial Sloan-Kettering Cancer Center (MSKCC) nomograms and those models with the addition of an mpMRI covariate to predict ECE [84]. Multivariate analysis revealed that mpMRI and PSA were significantly $(\mathrm{p}<0.001$ and $\mathrm{p}<0.048$, respectively) associated with the detection of ECE. Unlike previous studies [85,86], GS, clinical stage and percent positive cores were not significantly associated with detection of ECE $(\mathrm{p}=0.53, \mathrm{p}=0.645$ and $\mathrm{p}=0.08$, respectively). The AUC of the Partin tables was calculated as 0.85 and the MSKCC to be 0.86 . Adding an mpMRI parameter improved the Partin table AUC significantly $(0.93 ; p=0.017)$, while the MSKCC nomogram also increased significantly $(0.94 ; \mathrm{p}=0.023)$.

Further evidence in favor of an mpMRI nomogram for the prediction of ECE is provided by a retrospective analysis comparing mpMRI to 2001 Partin tables [38]. Interestingly, the authors developed a novel mpMRI staging system based on lesion suspicion, which corresponded to the TNM staging scheme [87]. Characteristics such as broad $(>12 \mathrm{~mm})$ tumor contact, capsular bulge, rectoprostatic angle obliteration and neurovascular bundle changes were utilized to determine what constituted ECE on imaging [88]. The AUC of the mpMRI nomogram was 0.82 , whereas the Partin table's nomogram AUC was significantly lower at $0.62(\mathrm{p}=0.04)$. Although there is no consensus regarding which clinicopathologic criteria are useful covariates in predicting ECE, these studies provides evidence that mpMRI findings increase the performance of nomograms, regardless of which parameters are used.

More recently Baco et al. evaluated mpMRI (T2W and DWI techniques) and determined tumor capsular contact length as compared with Partin tables for the prediction of ECE [89]. Similar to other studies [87], the mpMRI measured tumor contact length model AUC was 0.880 (95\% CI: 0.817-0.913), while the AUC 
of the Partin table model was 0.632 (95\% CI: 0.525-0.738). Importantly, as ECE on imaging is a subjective visual finding, this model improves upon a qualitative parameter by replacing it with a quantitative parameter, namely tumor contact length.

\section{- SVI}

External validation and comparison of the 1997 [74] and the 2001 Partin tables [75] for predicting SVI was performed by Augustin et al. [90] . The 2001 Partin table AUC was 0.775 (95\% CI: 0.746-0.804), while the 1997 Partin table AUC was 0.791 (95\% CI: 0.763-0.821). Similarly, in a validation study of the 2001 Partin tables, the AUC was found to be 0.74 [91]. PSA, clinical stage, biopsy GS and percentage positive core were all predictive for SVI [92]. ROC analysis of the percent positive biopsies alone produced an AUC of 0.80 , while the combination of all predictive covariates into a single model increased the AUC to only 0.84. Consequently, the addition of more classic clinicopathologic parameters to a model predicting SVI may not translate to increased utility. Rather than adding more classical parameters, further refined variables that account for anatomical correlates may be needed.

Staging with MRI has relied primarily on T2W imaging (T2WI). The main diagnostic criteria used to identify SVI on imaging were disruption in normal architecture and low-signal intensity, which can be evaluated using T2WI alone [93]. However, the first study to investigate the value of T2WI and DWI MRI sequences combined, when compared with T2WI alone, found the combined model to perform better [94]. Contrary to similar studies which found little benefit in using multiple MRI sequences for the detection of ECE [95], the AUC for the T2WI model was 0.82 , increasing significantly to 0.93 with the addition of DWI $(\mathrm{p}<0.01)$. This AUC was the highest reported to date, however staging MRI was performed on each patient for whom RP was considered. As such, the reported AUC values may be artificially inflated because patients were more likely to receive surgery if the mpMRI study was abnormal (verification bias). Furthermore, there may be inherent bias in adding MRI sequences, as these sequences cannot be truly separated at the time of image analysis, thus the reader is not truly blinded. However, this study does provide evidence for some usefulness of functional MRI sequences when compared with conventional MRI alone. Indeed, a recent study found that mpMRI suspicion score was statistically significant for predicting SVI $(p=0.04)$ and that lesions moderately or highly suspicious for SVI on mpMRI were confirmed on histopathology $71 \%$ of the time [96].

Another study investigated the added utility of mpMRI to a previously reported predictive model for SVI [97] by retrospectively analyzing patients who underwent mpMRI [98]. Previously described diagnostic criteria for SVI were used including seminal vesicle architectural changes (T2WI) and low signal intensity tissue changes (T2WI and DWI with ADC mapping) [93]. Multivariate analysis revealed MRI findings, PSA levels, Gleason grade and greatest percent cancer in biopsy cores were found to be significantly associated with SVI (range: $\mathrm{p}<0.0001$ to $\mathrm{p}<0.01)$. AUC was greatest for MRI alone (0.76) in comparison to any clinicopathological variables (0.62-0.73). The nomogram using only classical criteria generated an AUC of 0.80 , which was smaller than originally reported [97]. When the mpMRI parameter was included, the AUC increased significantly to $0.87(\mathrm{p}=0.04)$. As staging is dependent on the extent of cancer involved anatomy, the ability to visualize the anatomy has great utility.

\section{- LNI}

Studies have validated both the 1997 Partin table's [74] and 2001 Partin table's [99] ability to discriminate between those patients with and without LNI using models which combined clinical stage, Gleason sum and preoperative PSA [86,100]. They found that the 1997 Partin tables's AUC for predicting LNI was 0.818-0.837, while the 2001 tables AUC was 0.807 . Work was completed in an effort to compare the AUC of MRI findings alone to the 2001 Partin table nomogram, in patients undergoing standard pelvic lymph node dissection (pLND) after RP [101]. On multivariate analysis, significant predictors of LNI were MRI findings $(\mathrm{p}=0.002)$, PSA $(\mathrm{p}=0.004)$, greatest percent of cancer in all biopsy cores $(p=0.007)$ and GS ( $p=0.007)$. The AUC of the MRI findings alone was 0.633 . The Partin table nomogram with MRI findings as an added parameter generated an AUC of 0.892, which is higher than those reported by the Partin table validation studies [86,100].

More so than SVI, the addition of functional sequences, such as DWI, may have greater efficacy in the identification of those patients 


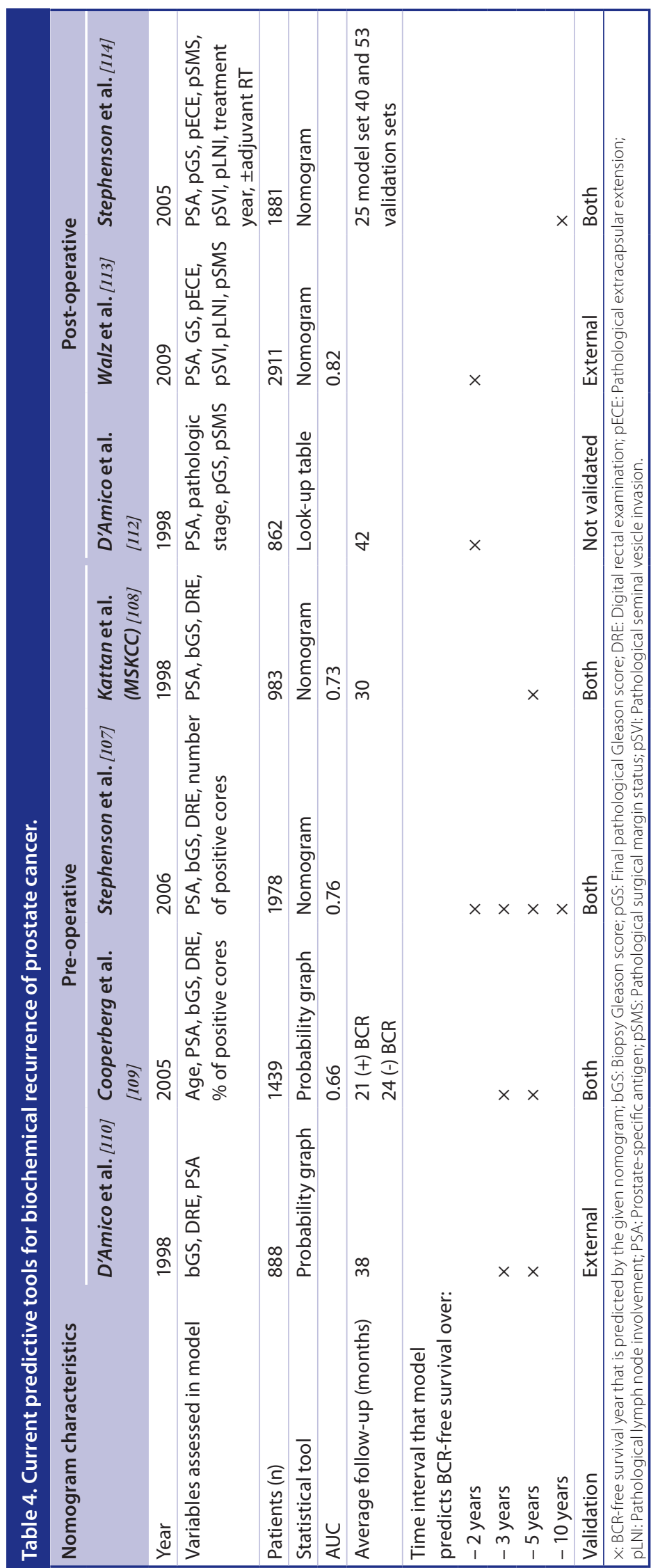

with LNI because morphological and intensity variations have been shown to be less prognostic $[102,103]$. One study investigated the utility of mpMRI (T2W, DCE and DWI) for predicting lymph node micrometastasis in patients who underwent standard pLND [104]. Patients were excluded if pelvic lymph nodes were greater than one centimeter on imaging. This exclusion criterion was created because it has been shown that $70 \%$ of LNI do not meet conventional size criteria on MRI T2WI alone [103]. Multivariate analysis of clinicopathologic and mpMRI findings revealed only mpMRI stage to be significant $(p=0.044)$. Receiver operator characteristic curve analysis showed that mpMRI stage yielded the highest AUC of 0.954 . This study may be limited by the use of standard pLND, which has been shown to underestimate the true extent of pelvic LNI when compared with extended lymph node dissection [105]. It does however highlight the utility of mpMRI when compared with clinicopathologic parameters, in predicting patients with microfocal lymph node metastasis. Furthermore, this study highlights that the addition of functional MRI sequences aids in the identification of LNI that may be missed on T2WI alone.

\section{Prediction of biochemical recurrence}

Given the recent introduction of mpMRI and the relatively prolonged natural history of $\mathrm{PCa}$, data are limited associating imaging findings with biochemical recurrence (BCR). BCR does however have a clear association with increased risk of metastatic disease and poorer PCa-specific mortality [106].

\section{- Predictive tools for $\mathrm{BCR}$}

Currently, the most commonly used preoperative predictive tools for BCR have been the Stephenson preoperative nomogram $(\mathrm{n}=1,978$, AUC: 0.706) [107], MSKCC nomogram ( $\mathrm{n}=983$; AUC: 0.73$)$ [108], CAPRA score ( $\mathrm{n}=1439$; AUC: 0.660) [109], and D'Amico et al. risk stratification ( $\mathrm{n}=888$; AUC: not reported) (Table 4) [110]. However, a comparative study of three of these predictive tools revealed the Stephenson and CAPRA models to have better discrimination than the D'Amico model [111]. The AUCs for 3 -year BCR was $0.735,0.742$ and 0.704 for the Stephenson, CAPRA and D'Amico models, respectively. The AUCs for 5-year BCR predictions were similar (Stephenson: 0.735, CAPRA: 0.729, D’Amico: 0.674). 
D'Amico et al. developed a table using clinicopathologic parameters to predict postoperative BCR [112]. The predictive parameters used in this table were preoperative PSA, pathologic stage, final GS and surgical margins. Walz et al. would later develop a more accurate predictive tool for $\mathrm{BCR}$ up to two years post-RP [113]. The variables included were preoperative PSA and final pathological findings (margins, GS, ECE, SVI and LNI). ROC analysis of this nomogram revealed an AUC of 0.82. Identifying patients at risk of early BCR could potentially inform the need for adjuvant androgen deprivation or radiotherapy. Stephenson's postoperative nomogram can predict $B C R$ up to 10 years post-RP and utilizes the same variables as the Walz nomogram (Table 4). This has been externally validated in two large patient cohorts $(\mathrm{n}=1782$ and $\mathrm{n}=1357)$ with AUCs of 0.79 and 0.81 , respectively [114].

\section{- Multiparametric MRI as a predictive tool for $B C R$}

There are few studies reporting on the use of mpMRI-based nomograms for the prediction of BCR. Initially, the role of MRI in predicting BCR was studied by Poulakis et al. using artificial neural networks [115]. However, these neural networks are not universally accepted because of a lack of transparency [116]. The addition of MRI findings, in combination with the clinicopathologic variables used in the MSKCC nomogram, showed significantly superior performance when compared with the MSKCC nomogram variables alone (AUC: 0.897 vs 0.765 ; $\mathrm{p}<0.05$ ). Furthermore, removing clinical stage as a parameter from the MSKCC nomogram did not affect performance (AUC: 0.897 vs 0.895 ).

In the largest study to date, mpMRI of 610 patients with biopsy-confirmed PCa were retrospectively scored on a seven-point scale $(0$ : no tumor seen to 7: LN metastasis). After controlling for potential confounders, increasing mpMRI score was found to be significantly associated with BCR (5-year hazard ratio: 1.76; $\mathrm{p}<0.0001 ; 10$-year hazard ratio: 1.81; $\mathrm{p}<0.0001)$ [117]. Additionally, the AUCs of 5 - and 10-year biochemical recurrence-free survival nomograms increased from 0.762 to 0.776 $(\mathrm{p}=0.081)$ and 0.773 to $0.788(\mathrm{p}=0.107)$, respectively, when mpMRI data were added [117]. An mpMRI with malignant characteristics (peripheral zone location, size $\geq 5 \mathrm{~mm}$ ) has also been shown to be an independent predictor of BCR [118]. Furthermore, Park et al. showed that

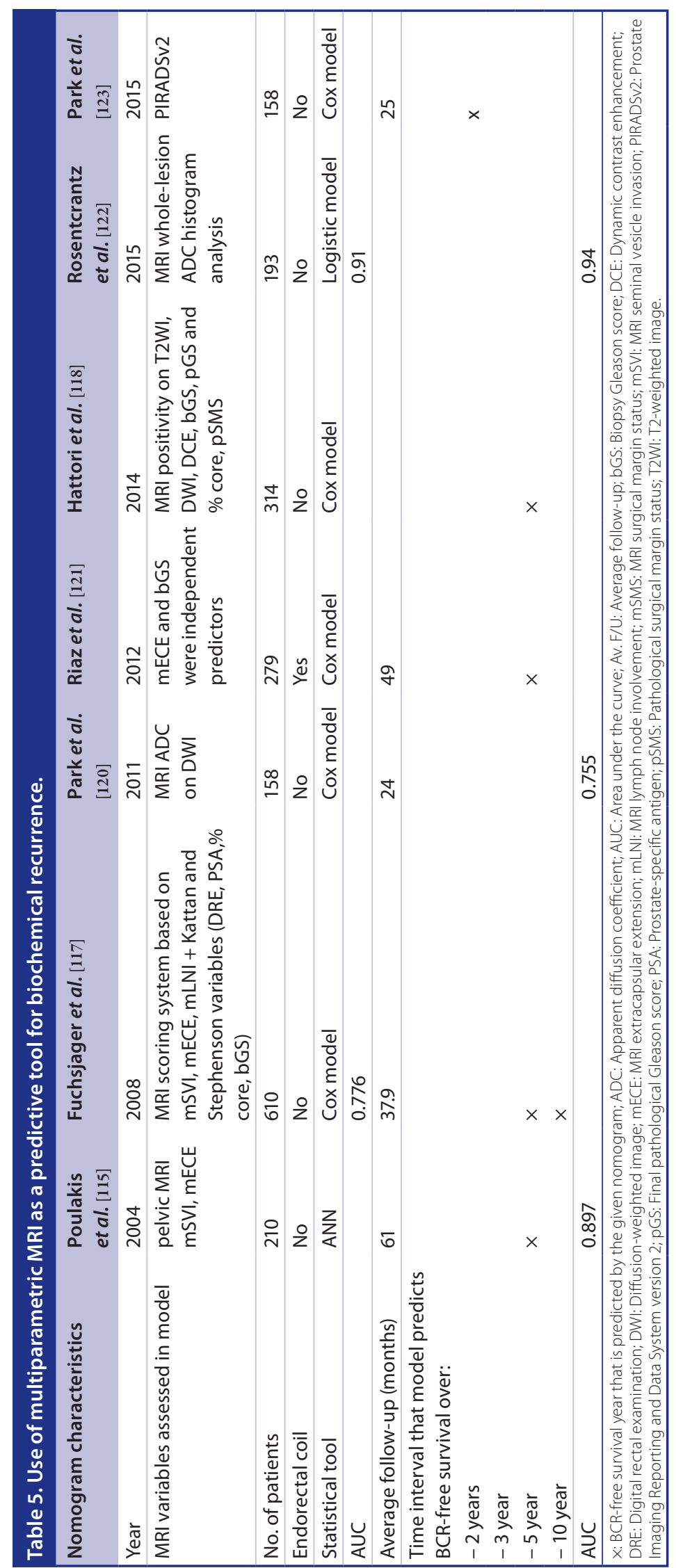


the combined presence of tumor on T2WI, DWI and DCE-MRI was an independent predictor of BCR (Table 5) [119]. Presently, there are no mpMRI based nomograms that have been developed and validated to predict BCR.

\section{Conclusion}

mpMRI has shown great improvement in predicting those at risk of $\mathrm{PCa}$, identifying $\mathrm{CS}$ disease, helping predict the extent of disease and in predicting disease progression. As a result of this promising evidence, mpMRI is now being integrated into the urologist's clinical practice. As
mpMRI continues to evolve, and is integrated with prognostic nomograms and novel biomarkers, its impact on the diagnosis, staging and management of PCa will only continue to increase.

Financial \& competing interests disclosure This research was supported by the Intramural Research Program of the NIH, National Cancer Institute, Center for Cancer Research and the Center for Interventional Oncology. NIH and Philips Healthcare have a cooperative research and development agreement. NIH and Philips share intellectual property in the field. This research was also made possible through the National Institutes of Health

\section{EXECUTIVE SUMMARY}

- Novel biomarkers such as PCA3, prostate health index and 4-Kallikrein score outperform total prostate-specific antigen in the screening of men for prostate cancer (PCa).

- The addition of multiparametric MRI (mpMRI) to novel biomarkers significantly improves PCa screening ability.

- Currently used risk calculators use patient specific clinicopathological parameters to calculate an individual's probability of being diagnosed with prostate cancer.

- Risk calculators may not accurately represent the predicted probability of prostate cancer in all patients.

- mpMRI outperforms commonly used risk calculators, such as the Prostate Cancer Prevention Trial Risk Calculator 2.0, for identifying men at high-risk prostate cancer diagnosis.

- Initial work examining the role of mpMRI in active surveillance (AS) has demonstrated its utility for confirming candidacy for AS and monitoring disease progression with serial mpMRIs.

- Additional studies are necessary to further validate the use of mpMRI along with strategic FBx as either a primary or adjunctive strategy for patients on AS.

- mpMRI may perform better than classical clinicopathologic parameters in predicting those patients with or without extracapsular extension.

- The addition of mpMRI as a covariate improves the predictive ability of classical nomograms for extracapsular extension (ECE).

- The prospect of mpMRI as a potential ECE nomogram parameter increases as imaging characteristics become less qualitative and more quantitative.

- The evidence in favor of which specific classic clinicopathological covariates are most useful in predicting seminal vesicle invasion ( $\mathrm{SVI})$ is mixed.

- Evidence reveals that the supplementation of currently used clinicopathologic parameters with further reiterations of these classic parameters adds minimal predictive value for SVI.

- The addition of the functional sequences used in mpMRI may be a value add to conventional MRI, which the evidence already reveals has predictive utility for SVI.

- MRI increases the ability of classic predictive tables to prognosticate lymph node involvement (LNI).

- MRI conventionally relies on size of lymph nodes to identify LNI, which can miss micrometastasis.

- The addition of quantitative mpMRI sequences may further improve MRI as a predictive parameter for LNI.

- Current gold standard nomograms perform well in predicting PCa biochemical recurrence (BCR).

- PCa diagnosis and management is changing as new technologies (mpMRI, MRI/US fusion biopsy, genomics, etc.) arise.

- mpMRI parameters alone and in combination with clinicopathological variables show better prediction of BCR.

- Highly powered studies are needed to elucidate the role of mpMRI as a predictor of BCR. 
Medical Research Scholars Program, a public-private partnership supported jointly by the NIH and generous contributions to the Foundation for the NIH from Pfizer Inc., The Doris Duke Charitable Foundation, The Alexandria Real Estate Equities, Inc. and Mr and Mrs Joel S Marcus and the Howard Hughes Medical Institute, as well as other private donors. For a complete list, please visit the Foundation website at: http://fnih.org/work/ education-training-0/medical-research-scholars-program. The authors have no other relevant affliations or financial involvement with any organization or entity with a financial interest in or financial conflict with the subject matter or materials discussed in the manuscript apart from those disclosed.

No writing assistance was utilized in the production of this manuscript.

\section{References}

1 Meigs JB, Barry MJ, Oesterling JE, Jacobsen SJ. Interpreting results of prostate-specific antigen testing for early detection of prostate cancer. J. Gen. Intern. Med. 11(9), 505-512 (1996).

2 Barry MJ. Clinical practice. Prostate-specificantigen testing for early diagnosis of prostate cancer. N. Engl. J. Med. 344(18), 1373-1377 (2001).

3 Loeb S, Bjurlin MA, Nicholson J et al. Overdiagnosis and overtreatment of prostate cancer. Eur. Urol. 65(6), 1046-1055 (2014).

4 Moyer VA. Screening for prostate cancer: U.S. Preventive Services Task Force recommendation statement. Ann. Int. Med. 157(2), 120-134 (2012).

5 Jemal A, Fedewa SA, Ma J et al. Prostate cancer incidence and PSA testing patterns in relation to USPSTF screening recommendations. JAMA 314(19), 2054-2061 (2015).

6 Hamoen EH, De Rooij M, Witjes JA, Barentsz JO, Rovers MM. Use of the Prostate Imaging Reporting and Data System (PI-RADS) for prostate cancer detection with multiparametric magnetic resonance imaging: a diagnostic meta-analysis. Eur. Urol. 67(6), 1112-1121 (2015).

7 Kongnyuy M, George AK, Rastinehad AR, Pinto PA. Magnetic resonance imagingultrasound fusion-guided prostate biopsy: review of technology, techniques, and outcomes. Curr. Urol. Rep. 17(4), 32 (2016).

8 Frye TP, Pinto PA, George AK. Optimizing patient population for MP-MRI and fusion biopsy for prostate cancer detection. Curr. Urol. Rep. 16(7), 50 (2015).

9 Rastinehad AR, Turkbey B, Salami SS et al. Improving detection of clinically significant prostate cancer: magnetic resonance imaging/transrectal ultrasound fusion guided prostate biopsy. J. Urol. 191(6), 1749-1754 (2014).

10 Turkbey B, Mani H, Shah V et al. Multiparametric 3T prostate magnetic resonance imaging to detect cancer: histopathological correlation using prostatectomy specimens processed in customized magnetic resonance imaging based molds. J. Urol. 186(5), 1818-1824 (2011).

11 Haffner J, Lemaitre L, Puech P et al. Role of magnetic resonance imaging before initial biopsy: comparison of magnetic resonance imaging-targeted and systematic biopsy for significant prostate cancer detection. BJU Int. 108(8 Pt 2), E171-E178 (2011).

12 Barentsz JO, Richenberg J, Clements R et al. ESUR prostate MR guidelines 2012. Eur. Radiol. 22(4), 746-757 (2012).

13 Rastinehad AR, Abboud SF, George AK et al. Reproducibility of multiparametric MRI and fusion-guided prostate biopsy: multiinstitutional external validation by a propensity score matched cohort. J. Urol. doi:10.1016/j.juro.12.102 (2016) (Epub ahead of print).

14 Rothwax JT, George AK, Wood BJ, Pinto PA. Multiparametric MRI in biopsy guidance for prostate cancer: fusion-guided. Biomed. Res. Int. 2014, 439171 (2014).

15 Siddiqui MM, Rais-Bahrami S, Turkbey B et al. Comparison of MR/ultrasound fusion-guided biopsy with ultrasound-guided biopsy for the diagnosis of prostate cancer. JAMA 313(4), 390-397 (2015).

16 Siddiqui MM, George AK, Rubin R et al. Efficiency of prostate cancer diagnosis by MR/ultrasound fusion-guided biopsy vs standard extended-sextant biopsy for MR-visible lesions. J. Natl Cancer Inst. 108(9), (2016).

17 Sankineni S, George AK, Brown AM et al. Posterior subcapsular prostate cancer: identification with mpMRI and MRI/TRUS fusion-guided biopsy. Abdom. Imaging 40 (7), 2557-2565 (2015).

18 Muller BG, Kaushal A, Sankineni S et al. Multiparametric magnetic resonance imaging-transrectal ultrasound fusion-assisted biopsy for the diagnosis of local recurrence after radical prostatectomy. Urol. Oncol. 33(10), 425.e421-426 (2015).
19 Wysock JS, Mendhiratta N, Zattoni F et al. Predictive value of negative $3 \mathrm{~T}$ multiparametric prostate MRI on 12 core biopsy results. BJU Int. doi:10.1111/bju.13427 (2016) (Epub ahead of print).

20 Filson CP, Natarajan S, Margolis DJ et al. Prostate cancer detection with magnetic resonance-ultrasound fusion biopsy: the role of systematic and targeted biopsies. Cancer 122(6), 884-892 (2016).

21 George AK, Turkbey B, Valayil SG et al. A urologist's perspective on prostate cancer imaging: past, present, and future. Abdom. Radiol. (New York) 41(5), 805-816 (2016).

22 Nam RK, Kattan MW, Chin JL et al. Prospective multi-institutional study evaluating the performance of prostate cancer risk calculators. J. Clin. Oncol. 29(22), 2959-2964 (2011).

23 Kranse R, Roobol M, Schroder FH. A graphical device to represent the outcomes of a logistic regression analysis. Prostate 68(15), 1674-1680 (2008).

24 Thompson IM, Ankerst DP, Chi C et al. Assessing prostate cancer risk: results from the Prostate Cancer Prevention Trial. J. Natl Cancer Inst. 98(8), 529-534 (2006).

25 Schroder F, Kattan MW. The comparability of models for predicting the risk of a positive prostate biopsy with prostate-specific antigen alone: a systematic review. Eur. Urol. 54(2), 274-290 (2008).

26 Caras RJ, Sterbis JR. Prostate cancer nomograms: a review of their use in cancer detection and treatment. Curr. Urol. Rep. 15(3), 391 (2014).

27 Thompson IM, Ankerst DP, Chi C et al. Operating characteristics of prostate-specific antigen in men with an initial PSA level of $3.0 \mathrm{ng} / \mathrm{ml}$ or lower. JAMA 294(1), 66-70 (2005).

28 Cui Y, Cao W, Li Q et al. Evaluation of prostate cancer antigen 3 for detecting prostate cancer: a systematic review and meta-analysis. Sci. Rep. 6, 25776 (2016).

29 Wang W, Wang M, Wang L, Adams TS, Tian $\mathrm{Y}, \mathrm{Xu}$ J. Diagnostic ability of\%p2PSA and 
prostate health index for aggressive prostate cancer: a meta-analysis. Sci. Rep. 4, 5012 (2014).

30 Parekh DJ, Punnen S, Sjoberg DD et al. A multi-institutional prospective trial in the USA confirms that the 4 Kscore accurately identifies men with high-grade prostate cancer. Eur. Urol. 68(3), 464-470 (2015).

31 Shakir NA, George AK, Siddiqui MM et al. Identification of threshold prostate specific antigen levels to optimize the detection of clinically significant prostate cancer by magnetic resonance imaging/ultrasound fusion guided biopsy. J. Urol. 192(6), 1642-1648 (2014).

32 Porpiglia F, Russo F, Manfredi M et al. The roles of multiparametric magnetic resonance imaging, PCA3 and prostate health index - which is the best predictor of prostate cancer after a negative biopsy? J. Urol. doi:10.1016/j.juro.01.030 (2014) (Epub ahead of print).

33 Porpiglia F, Cantiello F, De Luca S et al. In-parallel comparative evaluation between multiparametric magnetic resonance imaging, prostate cancer antigen 3 and the prostate health index in predicting pathologically confirmed significant prostate cancer in men eligible for active surveillance. BJU Int. doi:10.1111/bju.13318 (2015) (Epub ahead of print).

34 Kaufmann S, Bedke J, Gatidis S et al. Prostate cancer gene 3 (PCA3) is of additional predictive value in patients with PI-RADS grade III (intermediate) lesions in the MR-guided re-biopsy setting for prostate cancer. World J. Urol. 34(4), 509-515 (2016).

35 Leyten GH, Wierenga EA, Sedelaar JP et al. Value of PCA3 to predict biopsy outcome and its potential role in selecting patients for multiparametric MRI. Int. J. Mol. Sci. 14(6), 11347-11355 (2013).

36 Thompson IM, Goodman PJ, Tangen CM et al. The influence of finasteride on the development of prostate cancer. N. Engl. J. Med. 349 (3), 215-224 (2003).

37 Lundon DJ, Kelly BD, Foley R et al. Prostate cancer risk assessment tools in an unscreened population. World J. Urol. 33(6), 827-832 (2015).

38 Oliveira M, Marques V, Carvalho AP, Santos A. Head-to-head comparison of two online nomograms for prostate biopsy outcome prediction. BJU Int. 107(11), 1780-1783 (2011).

39 Trottier G, Roobol MJ, Lawrentschuk N et al. Comparison of risk calculators from the Prostate Cancer Prevention Trial and the European Randomized Study of Screening for
Prostate Cancer in a contemporary Canadian cohort. BJU Int. 108(8 Pt 2), E237-244 (2011).

40 Cavadas V, Osorio L, Sabell F, Teves F, Branco F, Silva-Ramos M. Prostate cancer prevention trial and European randomized study of screening for prostate cancer risk calculators: a performance comparison in a contemporary screened cohort. Eur. Urol. 58(4), 551-558 (2010).

41 Ankerst DP, Boeck A, Freedland SJ et al. Evaluating the Prostate Cancer Prevention Trial high grade prostate cancer risk calculator in 10 international biopsy cohorts: results from the Prostate Biopsy Collaborative Group. World J. Urol. 32(1), 185-191 (2014).

42 Ankerst DP, Boeck A, Freedland SJ et al. Evaluating the PCPT risk calculator in ten international biopsy cohorts: results from the Prostate Biopsy Collaborative Group. World J. Urol. 30 (2), 181-187 (2012).

43 Costa DN, Lotan Y, Rofsky NM et al. Assessment of prospectively assigned Likert scores for targeted magnetic resonance imaging-transrectal ultrasound fusion biopsies in patients with suspected prostate cancer. J. Urol. 195(1), 80-87 (2016).

44 Sonn GA, Chang E, Natarajan S et al. Value of targeted prostate biopsy using magnetic resonance-ultrasound fusion in men with prior negative biopsy and elevated prostatespecific antigen. Eur. Urol. 65(4), 809-815 (2014).

45 Rais-Bahrami S, Siddiqui MM, Turkbey B et al. Utility of multiparametric magnetic resonance imaging suspicion levels for detecting prostate cancer. J. Urol. 190(5), 1721-1727 (2013).

46 Abd-Alazeez M, Kirkham A, Ahmed HU et al. Performance of multiparametric MRI in men at risk of prostate cancer before the first biopsy: a paired validating cohort study using template prostate mapping biopsies as the reference standard. Prostate Cancer Prostatic Dis. 17(1), 40-46 (2014).

47 Kongnyuy M, Sidana A, George AK et al. The significance of anterior prostate lesions on multiparametric magnetic resonance imaging in African-American men. Urol. Oncol. doi:10.1016/j.urolonc.12.018 (2016) (Epub ahead of print).

48 Epstein JI, Walsh PC, Carmichael M, Brendler CB. Pathologic and clinical findings to predict tumor extent of nonpalpable (stage T1c) prostate cancer. JAMA 271(5), 368-374 (1994).

49 Bastian PJ, Mangold LA, Epstein JI, Partin AW. Characteristics of insignificant clinical
T1c prostate tumors. A contemporary analysis. Cancer 101(9), 2001-2005 (2004).

50 Salami SS, Vira MA, Turkbey B et al. Multiparametric magnetic resonance imaging outperforms the Prostate Cancer Prevention Trial risk calculator in predicting clinically significant prostate cancer. Cancer 120 (18), 2876-2882 (2014).

51 Fascelli M, Rais-Bahrami S, Sankineni S et al. Combined biparametric prostate magnetic resonance imaging and prostate-specific antigen in the detection of prostate cancer: a validation study in a biopsy-naive patient population. Urology 88 125-134 (2016).

52 Wilt TJ, Brawer MK, Jones KM et al. Radical prostatectomy versus observation for localized prostate cancer. N. Engl. J. Med. 367(3), 203-213 (2012).

53 Eggener SE, Badani K, Barocas DA et al. Gleason 6 prostate cancer: translating biology into population health. J. Urol. 194(3), 626-634 (2015).

54 Tosoian JJ, Carter HB, Lepor A, Loeb S. Active surveillance for prostate cancer: current evidence and contemporary state of practice. Nat. Rev. Urol. 13(4), 205-215 (2016).

55 George AK, Pinto PA, Rais-Bahrami S. Multiparametric MRI in the PSA screening era. Biomed. Res. Int. 2014, 465816 (2014).

56 Fascelli M, George AK, Frye T, Turkbey B, Choyke PL, Pinto PA. The role of MRI in active surveillance for prostate cancer. Curr. Urol. Rep. 16(6), 42 (2015).

57 George AK, Pinto PA. Editorial comment. Urology 85(2), 429 (2015).

58 Okoro C, George AK, Siddiqui MM et al. Magnetic resonance imaging/transrectal ultrasonography fusion prostate biopsy significantly outperforms systematic 12-Core biopsy for prediction of total magnetic resonance imaging tumor volume in active surveillance patients. J. Endourol. 29(10), 1115-1121 (2015).

59 Somford DM, Hamoen EH, Futterer JJ et al. The predictive value of endorectal 3 Tesla multiparametric magnetic resonance imaging for extraprostatic extension in patients with low, intermediate and high risk prostate cancer. J. Urol. 190(5), 1728-1734 (2013).

60 Yerram NK, Volkin D, Turkbey B et al. Low suspicion lesions on multiparametric magnetic resonance imaging predict for the absence of high-risk prostate cancer. BJU Int. 110 (11 Pt B), E783-788 (2012).

61 Schoots IG, Petrides N, Giganti F et al. Magnetic resonance imaging in active surveillance of prostate cancer: a systematic review. Eur. Urol. 67(4), 627-636 (2015). 
62 Turkbey B, Mani H, Aras O et al. Prostate cancer: can multiparametric MR imaging help identify patients who are candidates for active surveillance? Radiology 268(1), 144-152 (2013).

63 Hu JC, Chang E, Natarajan S et al. Targeted prostate biopsy in select men for active surveillance: do the Epstein criteria still apply? J. Urol. 192(2), 385-390 (2014).

64 Stamatakis L, Siddiqui MM, Nix JW et al. Accuracy of multiparametric magnetic resonance imaging in confirming eligibility for active surveillance for men with prostate cancer. Cancer 119(18), 3359-3366 (2013).

65 Da Rosa MR, Milot L, Sugar L et al. A prospective comparison of MRI-US fused targeted biopsy versus systematic ultrasoundguided biopsy for detecting clinically significant prostate cancer in patients on active surveillance. J. Magn. Reson. Imaging 41(1), 220-225 (2015).

66 Sonn GA, Filson CP, Chang E et al. Initial experience with electronic tracking of specific tumor sites in men undergoing active surveillance of prostate cancer. Urol. Oncol. 32(7), 952-957 (2014).

67 Turkbey B, Xu S, Kruecker J et al. Documenting the location of prostate biopsies with image fusion. BJU Int. 107(1), 53-57 (2011).

68 Chelluri R, Kilchevsky A, George AK et al. Prostate cancer diagnosis on repeat MRITRUS fusion biopsy of benign lesions: recommendations for repeat sampling. J. Urol. doi:10.1016/j.juro.02.066 (2016) (Epub ahead of print).

69 Raskolnikov D, Rais-Bahrami S, George AK et al. The role of image guided biopsy targeting in patients with atypical small acinar proliferation. J. Urol. 193(2), 473-478 (2015).

70 Walton Diaz A, Shakir NA, George AK et al. Use of serial multiparametric magnetic resonance imaging in the management of patients with prostate cancer on active surveillance. Urol. Oncol. 33(5), 202.e201-207 (2015).

71 Muller BG, Shih JH, Sankineni S et al. Prostate cancer: interobserver agreement and accuracy with the revised prostate imaging reporting and data system at multiparametric MR imaging. Radiology 277(3), 741-750 (2015).

72 Thompson I, Thrasher JB, Aus G et al. Guideline for the management of clinically localized prostate cancer: 2007 update. J. Urol. 177(6), 2106-2131 (2007).

73 Partin AW, Yoo J, Carter HB et al. The use of prostate specific antigen, clinical stage and
Gleason score to predict pathological stage in men with localized prostate cancer. J. Urol. 150(1), 110-114 (1993).

74 Partin AW, Kattan MW, Subong EN et al. Combination of prostate-specific antigen, clinical stage, and Gleason score to predict pathological stage of localized prostate cancer. A multi-institutional update. JAMA 277(18), 1445-1451 (1997).

75 Partin AW, Mangold LA, Lamm DM, Walsh PC, Epstein JI, Pearson JD. Contemporary update of prostate cancer staging nomograms (Partin tables) for the new millennium. Urology 58(6), 843-848 (2001).

76 Brockman JA, Alanee S, Vickers AJ et al. Nomogram predicting prostate cancerspecific mortality for men with biochemical recurrence after radical prostatectomy. Eur. Urol. 67(6), 1160-1167 (2015).

77 Wheeler TM, Dillioglugil O, Kattan MW et al. Clinical and pathological significance of the level and extent of capsular invasion in clinical stage T1-2 prostate cancer. Hum. Pathol. 29(8), 856-862 (1998).

78 Hull GW, Rabbani F, Abbas F, Wheeler TM, Kattan MW, Scardino PT. Cancer control with radical prostatectomy alone in 1,000 consecutive patients. J. Urol. 167(2 Pt 1), 528-534 (2002).

79 Pak S, Park S, Ryu J et al. Preoperative factors predictive of posterolateral extracapsular extension after radical prostatectomy. Korean J. Urol. 54(12), 824-829 (2013).

80 Partin AW, Borland RN, Epstein JI, Brendler $\mathrm{CB}$. Influence of wide excision of the neurovascular bundle(s) on prognosis in men with clinically localized prostate cancer with established capsular penetration. J. Urol. 150(1), 142-146; discussion 146-148 (1993).

81 Roethke MC, Lichy MP, Kniess M et al. Accuracy of preoperative endorectal MRI in predicting extracapsular extension and influence on neurovascular bundle sparing in radical prostatectomy. World J. Urol. 31(5), 1111-1116 (2013).

82 Raskolnikov D, George AK, Rais-Bahrami S et al. The role of magnetic resonance image guided prostate biopsy in stratifying men for risk of extracapsular extension at radical prostatectomy. J. Urol. 194(1), 105-111 (2015).

83 Wang L, Mullerad M, Chen HN et al. Prostate cancer: incremental value of endorectal MR imaging findings for prediction of extracapsular extension. Radiology 232(1), 133-139 (2004).
84 Feng TS, Sharif-Afshar AR, Wu J et al. Multiparametric MRI improves accuracy of clinical nomograms for predicting extracapsular extension of prostate cancer. Urology 86(2), 332-337 (2015).

85 Ohori M, Kattan MW, Koh H et al. Predicting the presence and side of extracapsular extension: a nomogram for staging prostate cancer. J. Urol. 171(5), 1844-1849; discussion 1849 (2004).

86 Graefen M, Augustin H, Karakiewicz PI et al. Can predictive models for prostate cancer patients derived in the United States of America be utilized in European patients? A validation study of the Partin tables. Eur. Urol. 43(1), 6-10; discussion 11 (2003).

87 Gupta RT, Faridi KF, Singh AA et al. Comparing 3-T multiparametric MRI and the Partin tables to predict organ-confined prostate cancer after radical prostatectomy. Urol. Oncol. 32(8), 1292-1299 (2014).

88 Yu KK, Hricak H, Alagappan R, Chernoff DM, Bacchetti P, Zaloudek CJ. Detection of extracapsular extension of prostate carcinoma with endorectal and phased-array coil MR imaging: multivariate feature analysis. Radiology 202(3), 697-702 (1997).

89 Baco E, Rud E, Vlatkovic L et al. Predictive value of magnetic resonance imaging determined tumor contact length for extracapsular extension of prostate cancer. J. Urol. 193(2), 466-472 (2015).

90 Augustin $\mathrm{H}$, Eggert T, Wenske $\mathrm{S}$ et al. Comparison of accuracy between the Partin tables of 1997 and 2001 to predict final pathological stage in clinically localized prostate cancer. J. Urol. 171(1), 177-181 (2004).

91 Yu JB, Makarov DV, Sharma R, Peschel RE, Partin AW, Gross CP. Validation of the partin nomogram for prostate cancer in a national sample. J. Urol. 183(1), 105-111 (2010).

92 Guzzo TJ, Vira M, Wang Y et al. Preoperative parameters, including percent positive biopsy, in predicting seminal vesicle involvement in patients with prostate cancer. J. Urol. 175(2), 518-522 (2006).

93 Sala E, Akin O, Moskowitz CS et al. Endorectal MR imaging in the evaluation of seminal vesicle invasion: diagnostic accuracy and multivariate feature analysis. Radiology 238(3), 929-937 (2006).

94 Kim CK, Choi D, Park BK, Kwon GY, Lim HK. Diffusion-weighted MR imaging for the evaluation of seminal vesicle invasion in prostate cancer: initial results. J. Magn. Reson. Imaging 28(4), 963-969 (2008). 
95 Soylu FN, Peng Y, Jiang Y et al. Seminal vesicle invasion in prostate cancer: evaluation by using multiparametric endorectal MR imaging. Radiology 267(3), 797-806 (2013).

96 Raskolnikov D, George AK, Rais-Bahrami S et al. Multiparametric magnetic resonance imaging and image-guided biopsy to detect seminal vesicle invasion by prostate cancer. J. Endourol. 28(11), 1283-1289 (2014).

97 Koh H, Kattan MW, Scardino PT et al. A nomogram to predict seminal vesicle invasion by the extent and location of cancer in systematic biopsy results. J. Urol. 170 (4 Pt 1), 1203-1208 (2003).

98 Wang L, Hricak H, Kattan MW et al. Prediction of seminal vesicle invasion in prostate cancer: incremental value of adding endorectal MR imaging to the Kattan nomogram. Radiology 242(1), 182-188 (2007).

99 Blute ML, Bergstralh EJ, Partin AW et al. Validation of Partin tables for predicting pathological stage of clinically localized prostate cancer. J. Urol. 164(5), 1591-1595 (2000).

100 Eifler JB, Feng Z, Lin BM et al. An updated prostate cancer staging nomogram (Partin tables) based on cases from 2006 to 2011. BJU Int. 111(1), 22-29 (2013).

101 Wang L, Hricak H, Kattan MW et al. Combined endorectal and phased-array MRI in the prediction of pelvic lymph node metastasis in prostate cancer. AJR Am. J. Roentgenol. 186(3), 743-748 (2006).

102 Giannarini G, Petralia G, Thoeny HC. Potential and limitations of diffusionweighted magnetic resonance imaging in kidney, prostate, and bladder cancer including pelvic lymph node staging: a critical analysis of the literature. Eur. Urol. 61(2), 326-340 (2012).

103 Hovels AM, Heesakkers RA, Adang EM et al. The diagnostic accuracy of CT and MRI in the staging of pelvic lymph nodes in patients with prostate cancer: a meta-analysis. Clin. Radiol. 63(4), 387-395 (2008).

104 Park SY, Oh YT, Jung DC, Cho NH, Choi YD, Rha KH. Prediction of Micrometastasis $(<1 \mathrm{~cm})$ to Pelvic Lymph Nodes in Prostate Cancer: Role of Preoperative MRI. AJR Am. J. Roentgenol. 205(3), W328-W334 (2015).

105 Bivalacqua TJ, Pierorazio PM, Gorin MA, Allaf ME, Carter HB, Walsh PC. Anatomic extent of pelvic lymph node dissection: impact on long-term cancer-specific outcomes in men with positive lymph nodes at time of radical prostatectomy. Urology 82(3), 653-658 (2013).

106 Freedland SJ, Humphreys EB, Mangold LA, Eisenberger M, Partin AW. Time to prostate specific antigen recurrence after radical prostatectomy and risk of prostate cancer specific mortality. J. Urol. $176(4 \mathrm{Pt} 1)$, 1404-1408 (2006).

107 Stephenson AJ, Scardino PT, Eastham JA et al. Preoperative nomogram predicting the 10 -year probability of prostate cancer recurrence after radical prostatectomy. J. Natl Cancer Inst. 98(10), 715-717 (2006).

108 Kattan MW, Eastham JA, Stapleton AM, Wheeler TM, Scardino PT. A preoperative nomogram for disease recurrence following radical prostatectomy for prostate cancer. J. Natl Cancer Inst. 90 (10), 766-771 (1998).

109 Cooperberg MR, Pasta DJ, Elkin EP et al. The University of California, San Francisco Cancer of the Prostate Risk Assessment score: a straightforward and reliable preoperative predictor of disease recurrence after radical prostatectomy. J. Urol. 173(6), 1938-1942 (2005).

110 D'Amico AV, Whittington R, Malkowicz SB et al. Biochemical outcome after radical prostatectomy, external beam radiation therapy, or interstitial radiation therapy for clinically localized prostate cancer. JAMA 280(11), 969-974 (1998).

111 Lughezzani G, Budaus L, Isbarn $\mathrm{H}$ et al. Head-to-head comparison of the three most commonly used preoperative models for prediction of biochemical recurrence after radical prostatectomy. Eur. Urol. 57(4), 562-568 (2010).

112 D'Amico AV, Whittington R, Malkowicz SB et al. The combination of preoperative prostate specific antigen and postoperative pathological findings to predict prostate specific antigen outcome in clinically localized prostate cancer. J. Urol. 160(6 Pt 1), 2096-2101 (1998).

113 Walz J, Chun FK, Klein EA et al. Nomogram predicting the probability of early recurrence after radical prostatectomy for prostate cancer. J. Urol. 181(2), 601-607; discussion 607-608 (2009).

114 Stephenson AJ, Scardino PT, Eastham JA et al. Postoperative nomogram predicting the 10 -year probability of prostate cancer recurrence after radical prostatectomy. J. Clin. Oncol. 23(28), 7005-7012 (2005).
115 Poulakis V, Witzsch U, De Vries R et al. Preoperative neural network using combined magnetic resonance imaging variables, prostate-specific antigen, and gleason score for predicting prostate cancer biochemical recurrence after radical prostatectomy. Urology 64(6), 1165-1170 (2004).

116 Abbod MF, Catto JW, Linkens DA, Hamdy FC. Application of artificial intelligence to the management of urological cancer. J. Urol. 178(4 Pt 1), 1150-1156 (2007).

117 Fuchsjager MH, Shukla-Dave A, Hricak H et al. Magnetic resonance imaging in the prediction of biochemical recurrence of prostate cancer after radical prostatectomy. BJU Int. 104(3), 315-320 (2009).

118 Hattori S, Kosaka T, Mizuno R et al. Prognostic value of preoperative multiparametric magnetic resonance imaging (MRI) for predicting biochemical recurrence after radical prostatectomy. BJU Int. 113(5), 741-747 (2014).

119 Park JJ, Kim CK, Park SY, Park BK, Lee HM, Cho SW. Prostate cancer: role of pretreatment multiparametric 3-T MRI in predicting biochemical recurrence after radical prostatectomy. AJR Am. J. Roentgenol. 202(5), W459-W465 (2014).

120 Park SY, Kim CK, Park BK, Lee HM, Lee KS. Prediction of biochemical recurrence following radical prostatectomy in men with prostate cancer by diffusion-weighted magnetic resonance imaging: initial results. Eur. Radiol. 21(5), 1111-1118 (2011).

121 Riaz N, Afaq A, Akin O et al. Pretreatment endorectal coil magnetic resonance imaging findings predict biochemical tumor control in prostate cancer patients treated with combination brachytherapy and externalbeam radiotherapy. Int. J. Radiat. Oncol. Biol. Phys. 84(3), 707-711 (2012).

122 Rosenkrantz AB, Ream JM, Nolan P, Rusinek H, Deng FM, Taneja SS. Prostate cancer: utility of whole-lesion apparent diffusion coefficient metrics for prediction of biochemical recurrence after radical prostatectomy. AJR Am. J. Roentgenol. 205(6), 1208-1214 (2015).

123 Park SY, Oh YT, Jung DC et al. Prediction of biochemical recurrence after radical prostatectomy with PI-RADS version 2 in prostate cancers: initial results. Eur Radiol. 26(8), 2502-2509 (2016). 